\title{
GROUP CLASSIFICATION AND SYMMETRY REDUCTION OF THREE-DIMENSIONAL NONLINEAR ANOMALOUS DIFFUSION EQUATION
}

\author{
R.K. GAZIZOV, A.A. KASATKIN, S.YU. LUKASHCHUK
}

\begin{abstract}
The work is devoted to studying symmetry properties of a nonlinear anomalous diffusion equation involving a Riemann-Liouville fractional derivative with respect to the time. We resolve a problem on group classification with respect to the diffusion coefficient treated as a function of the unknown. We show that for an arbitrary function, the equation admits a seven-dimensional Lie algebra of infinitesimal operators corresponding to the groups of translations, rotations and dilations. In contrast to the symmetries of the equations with integer order derivatives, the translation in time is not admitted. Moreover, the coefficients of the group of dilations are different. If the coefficient is power, the admissible algebra is extended to a eight-dimensional one by an additional operator generating the group of dilatations. For two specific values of the exponent in the power, the algebra can be further extended to a nine-dimensional one or to a eleven-dimensional one and at that, additional admissible operators correspond to various projective transformations. For the obtained Lie algebras of symmetries with dimensions from seven to nine, we construct optimal systems of subalgebras and provide ansatzes for corresponding invariant solutions of various ranks. We also provide general forms of invariant solutions convenient for the symmetry reduction as the fractional Riemann-Liouville derivative is present. We make a symmetry reduction on subalgebras allowing one to find invariant solutions of rank one. We provide corresponding reduced ordinary fractional differential equations.
\end{abstract}

Keywords: fractional derivatives, symmetry reduction, optimal system of subalgebras, nonlinear fractional diffusion equation.

Mathematics Subject Classification: 35R11, 35B06, 76M60

\section{INTRODUCTION}

Many modern mathematical models employ the techniques of fractional integration and differentiation for describing the memory effects or nonlocal interactions in a considered media [1], [2], [3]. In particular, under certain conditions, the fractional order derivatives can be applicable for the models of filtration in a soil or in complex oil and gas reservoirs. Many of the considered models, both classical and fractional-differential, involve essential nonlinearities in the equations [4], [5]. Almost all existing methods for the equations with fractional derivatives are either numerical or approximate analytic. One of the most developed direction in studying of nonlinear equations is the studying of their symmetry properties [6], [7]. These methods can be also adapted for studying equations with fractional order derivatives, see, for instance, survey [8].

R.K. Gazizov, A.A. Kasatkin, S.Yu. Lukashchuk, Group classification and symmetry reducTION OF THREE-DIMENSIONAL NONLINEAR ANOMALOUS DIFFUSION EQUATION.

(c) Gazizov R.K., Kasatkin A.A., Lukashchuk S.Yu. 2019.

The work is supported within project no. 1.3103.2017/4.6 of State Task of Ministery of Education and Science of Russian Federation.

Submitted November 18, 2019. 
In the present work we consider a three-dimensional nonlinear anomalous diffusion equation with a fractional derivative in time of the form

$$
{ }_{0} D_{t}^{\alpha} u=\left(k(u) u_{x}\right)_{x}+\left(k(u) u_{y}\right)_{y}+\left(k(u) u_{z}\right)_{z}, \quad \alpha \in(0,1) \cup(1,2) .
$$

In particular, such equation can be obtained while considering the filtration in a porous media with a modified Darcy law $[9]$.

A fractional derivative of Riemann-Liouville type is defined by the identity

$$
{ }_{a} D_{t}^{\alpha} u(t, \mathbf{x})=\frac{1}{\Gamma(m-\alpha)} \frac{d^{m}}{d t^{m}} \int_{a}^{t} \frac{u(w, \mathbf{x})}{(t-w)^{\alpha+1-m}} d t, \quad m-1<\alpha<m, \quad m \in \mathbb{N} .
$$

For equation (1) we solve the problem of a group classification with respect to the function $k(u)$. In the limiting case $\alpha=1$, by the identity ${ }_{0} D_{t}^{1} u=u_{t}$, this equation becomes a classical nonlinear heat equation, and the results of the group classification for the latter equation are well-known [10], see also [11]. The results on the group classification for one-dimensional version of equation (11) with symmetry reductions were provided in [12]. In the present work we consider only nonlinear equations assuming that $k^{\prime}(u) \neq 0$. Symmetry properties and conservations laws for equation (1) were considered in work [13], however, an essential part of the obtained results was wrong and the problem on the group classification (1) is still topical.

For the obtained Lie algebras of the symmetries of dimensions from seven to nine we construct optimal systems of subalgebras and find the substitutions for constructing invariant solutions. We consider the cases of symmetry reductions for invariant solutions of rank 1.

\section{GRoup CLASSIFICATION OF NONLINEAR ISOTROPIC FRACTIONAL DIFFERENTIAL ANOMALOUS DIFFUSION EQUATION}

The problem on group classification for equation (1) is solved up to equivalence transformations [6], [7], which can be found similar to symmetries [14] and read as

$$
\bar{t}=\delta^{2} t, \quad \bar{x}=\gamma x+\beta_{1}, \quad \bar{y}=\gamma y+\beta_{2}, \quad \bar{z}=\gamma z+\beta_{3}, \quad \bar{u}=\rho u, \quad \bar{k}=\gamma^{2} \delta^{-2 \alpha} k,
$$

where $\beta_{1}, \beta_{2}, \beta_{3}, \gamma, \delta \in \mathbb{R}, \rho>0$. We observe an important feature: opposite to the classical heat equation, the equivalence transformations for the fractional equation do not involve the translation in the variable $u$.

The symmetries of equation (1) are defined by the infinitesimal generators

$$
X=\tau \frac{\partial}{\partial t}+\xi^{1} \frac{\partial}{\partial x}+\xi^{2} \frac{\partial}{\partial y}+\xi^{3} \frac{\partial}{\partial z}+\eta \frac{\partial}{\partial u}
$$

and are sought in a linear autonomous form [8], [15]:

$$
\begin{array}{lll}
\tau=C_{1} t+C_{2} t^{2}, & \xi^{1}=\theta^{1}(x, y, z), & \xi^{2}=\theta^{2}(x, y, z), \quad \xi^{3}=\theta^{3}(x, y, z), \\
\eta=\eta_{(0)}+\eta_{(1)} u, & \eta_{(0)}=\psi(t, x, y, z), & \eta_{(1)}=\varphi(x, y, z)+(\alpha-1) C_{2} t
\end{array}
$$

and here we apply the prolongation formula to fractional derivatives.

The determining equation for the functions $\theta^{i},(i=1,2,3), \varphi$ and $\psi$ becomes

$$
\begin{aligned}
{ }_{0} D_{t}^{\alpha}(\psi) & +\left[\varphi-\alpha C_{1}-(1+\alpha) C_{2} t\right]\left[\left(u_{x x}+u_{y y}+u_{z z}\right) k+\left(u_{x}^{2}+u_{y}^{2}+u_{z}^{2}\right) k^{\prime}\right] \\
& -\left(\zeta_{11}+\zeta_{22}+\zeta_{33}\right) k-2\left(\zeta_{1} u_{x}+\zeta_{2} u_{y}+\zeta_{3} u_{z}\right) k^{\prime} \\
& -\left[\psi+\left(\varphi+(\alpha-1) C_{2} t\right) u\right]\left[\left(u_{x x}+u_{y y}+u_{z z}\right) k^{\prime}+\left(u_{x}^{2}+u_{y}^{2}+u_{z}^{2}\right) k^{\prime \prime}\right]=0
\end{aligned}
$$


where

$$
\begin{aligned}
\zeta_{1}= & \psi_{x}+\varphi_{x} u+\left(\varphi+(\alpha-1) C_{2} t-\theta_{x}^{1}\right) u_{x}-\theta_{x}^{2} u_{y}-\theta_{x}^{3} u_{z}, \\
\zeta_{2}= & \psi_{y}+\varphi_{y} u-\theta_{y}^{1} u_{x}+\left(\varphi+(\alpha-1) C_{2} t-\theta_{y}^{2}\right) u_{y}-\theta_{y}^{3} u_{z}, \\
\zeta_{3}= & \psi_{z}+\varphi_{z} u-\theta_{z}^{1} u_{x}-\theta_{z}^{2} u_{y}+\left(\varphi+(\alpha-1) C_{2} t-\theta_{z}^{3}\right) u_{z}, \\
\zeta_{11}= & \psi_{x x}+\varphi_{x x} u+\left(2 \varphi_{x}-\theta_{x x}^{1}\right) u_{x}-\theta_{x x}^{2} u_{y}-\theta_{x x}^{3} u_{z}+ \\
& +\left(\varphi+(\alpha-1) C_{2} t-2 \theta_{x}^{1}\right) u_{x x}-2 \theta_{x}^{2} u_{x y}-2 \theta_{x}^{3} u_{x z}, \\
\zeta_{22}= & \psi_{y y}+\varphi_{y y} u-\theta_{y y}^{1} u_{x}+\left(2 \varphi_{y}-\theta_{y y}^{2}\right) u_{y}-\theta_{y y}^{3} u_{z} \\
& -2 \theta_{y}^{1} u_{x y}+\left(\varphi+(\alpha-1) C_{2} t-2 \theta_{y}^{2}\right) u_{y y}-2 \theta_{y}^{3} u_{y z}, \\
\zeta_{33}= & \psi_{z z}+\varphi_{z z} u-\theta_{z z}^{1} u_{x}-\theta_{z z}^{2} u_{y}+\left(2 \varphi_{z}-\theta_{z z}^{2}\right) u_{z} \\
& -2 \theta_{z}^{2} u_{y z}+\left(\varphi+(\alpha-1) C_{2} t-2 \theta_{z}^{3}\right) u_{z z} .
\end{aligned}
$$

By splitting (6) with respect to $u_{x x}, u_{y y}$ and $u_{z z}$ we arrive at the equations

$$
\begin{aligned}
& \psi+(\alpha-1) C_{2} t u+\varphi u+\left[\alpha\left(C_{1}+2 C_{2} t\right)-2 \theta_{x}^{1}\right] K(u)=0, \\
& \psi+(\alpha-1) C_{2} t u+\varphi u+\left[\alpha\left(C_{1}+2 C_{2} t\right)-2 \theta_{y}^{2}\right] K(u)=0, \\
& \psi+(\alpha-1) C_{2} t u+\varphi u+\left[\alpha\left(C_{1}+2 C_{2} t\right)-2 \theta_{z}^{3}\right] K(u)=0,
\end{aligned}
$$

where $K(u)=k(u) / k^{\prime}(u)$. By differentiating these equations with respect to $u$ we are led to the classifying relation $K^{\prime \prime}=0$, which turns out to be identical to the classifying relation for classical heat equation [6]. As a result, up to equivalent transformations (3), we find the following cases for $k(u) \neq$ const:

I. $k(u)$ is an arbitrary function,

II. $k(u)=e^{u}$,

III. $k(u)=(u+B)^{\sigma}, \sigma \neq 0$.

In Case I, by (7) we find

$$
C_{2}=0, \quad \varphi=0, \quad \psi=0, \quad \theta_{x}^{1}=\theta_{y}^{2}=\theta_{z}^{3}=\frac{\alpha}{2} C_{1} .
$$

In Case II we have

$$
C_{2}=0, \quad \varphi=0, \quad \theta_{x}^{1}=\theta_{y}^{2}=\theta_{z}^{3}=\frac{\alpha}{2}\left(\alpha C_{1}+\psi\right) .
$$

In Case III we get the equations

$$
\sigma \psi=B\left(\sigma \varphi-2 \alpha C_{2} t\right), \quad \theta_{x}^{1}=\theta_{y}^{2}=\theta_{z}^{3}=\frac{\alpha}{2}\left(\alpha C_{1}+\psi\right),
$$

at that $C_{2} \neq 0$ only as $\sigma=\frac{2 \alpha}{1-\alpha}$.

The splitting of (6) with respect to the mixed variables $u_{x y}, u_{x z}$ and $u_{y z}$ gives rise to the equations

$$
\theta_{x}^{2}+\theta_{y}^{1}=0, \quad \theta_{x}^{3}+\theta_{z}^{1}=0, \quad \theta_{y}^{3}+\theta_{z}^{2}=0 .
$$

The splitting with respect to $u_{x}^{2}, u_{y}^{2}, u_{z}^{2}, u_{x} u_{y}, u_{x} u_{z}, u_{y} u_{z}$ produces the differential implications of the above obtained equations.

The splitting with respect to $u_{x}, u_{y}, u_{z}$ gives

$$
\begin{aligned}
& K(u)\left(2 \varphi_{x}-\theta_{x x}^{1}-\theta_{y y}^{1}-\theta_{z z}^{1}\right)+2\left(\psi_{x}+\varphi_{x} u\right)=0, \\
& K(u)\left(2 \varphi_{y}-\theta_{x x}^{2}-\theta_{y y}^{2}-\theta_{z z}^{2}\right)+2\left(\psi_{y}+\varphi_{y} u\right)=0, \\
& K(u)\left(2 \varphi_{z}-\theta_{x x}^{3}-\theta_{y y}^{3}-\theta_{z z}^{3}\right)+2\left(\psi_{z}+\varphi_{z} u\right)=0 .
\end{aligned}
$$

The equation remaining after the splitting reads as

$$
{ }_{0} D_{t}^{\alpha} \psi=\left(\psi_{x x}+\psi_{y y}+\psi_{z z}\right) k+\left(\varphi_{x x}+\varphi_{y y}+\varphi_{z z}\right) u k .
$$


The group classification is completed by finding the solution to system of obtained equations (8)-(13). At that, by straightforward calculations we show that Case II does not extend the group of point transformations admitted by equation (1), while in Case III the group extends only as $B=0$, and here one more particular $k(u)=u^{-\frac{4}{5}}$ arises. We summarize the obtained results in the following statement.

Lemma 1. Assuming that $k^{\prime}(u) \neq 0$, in the case of an arbitrary function $k(u)$ nonlinear equation (1) possesses a seven-dimensional Lie algebra of point symmetries with the basis

$$
\begin{aligned}
& X_{1}=\frac{\partial}{\partial x}, \quad X_{2}=\frac{\partial}{\partial y}, \quad X_{3}=\frac{\partial}{\partial z}, \quad X_{4}=y \frac{\partial}{\partial z}-z \frac{\partial}{\partial y}, \\
& X_{5}=z \frac{\partial}{\partial x}-x \frac{\partial}{\partial z}, \quad X_{6}=-y \frac{\partial}{\partial x}+x \frac{\partial}{\partial y}, \quad X_{7}=\frac{2}{\alpha} t \frac{\partial}{\partial t}+x \frac{\partial}{\partial x}+y \frac{\partial}{\partial y}+z \frac{\partial}{\partial z} .
\end{aligned}
$$

In the case of a power dependence $k(u)=u^{\sigma}, \sigma \neq 0$, the algebra is extended to an eightdimensional one by the operator

$$
X_{8}=u \frac{\partial}{\partial u}-\frac{\sigma}{\alpha} t \frac{\partial}{\partial t} .
$$

At that, in the particular case $\sigma=\frac{2 \alpha}{1-\alpha}$, there is an additional extension of the algebra to a nine-dimensional one with the operator

$$
X_{9}=t^{2} \frac{\partial}{\partial t}-(1-\alpha) t u \frac{\partial}{\partial u}
$$

and in the case $\sigma=-\frac{4}{5}$ the extension is made up to an eleven-dimensional algebra with the operators

$$
\begin{aligned}
& X_{9}=\left(y^{2}+z^{2}-x^{2}\right) \frac{\partial}{\partial x}-2 x y \frac{\partial}{\partial y}-2 x z \frac{\partial}{\partial z}+5 x u \frac{\partial}{\partial u}, \\
& X_{10}=\left(x^{2}+z^{2}-y^{2}\right) \frac{\partial}{\partial y}-2 x y \frac{\partial}{\partial x}-2 y z \frac{\partial}{\partial z}+5 y u \frac{\partial}{\partial u}, \\
& X_{11}=\left(x^{2}+y^{2}-z^{2}\right) \frac{\partial}{\partial z}-2 x z \frac{\partial}{\partial x}-2 y z \frac{\partial}{\partial y}+5 z u \frac{\partial}{\partial u} .
\end{aligned}
$$

There are no other cases for the considered nonlinear equation when the Lie algebra of symmetries extends.

Comparing the results of the performed classification with the classification results of the classical three-dimensional nonlinear heat equation $[6]$, we see that they are very close. The presence of the fractional derivative just changes the coefficients in the dilation operators $X_{7}$, $X_{8}$. However, the dimension of the admissible Lie algebra of symmetries for the fractional differential equations becomes less by one since the translation in time is not admitted. At that, we find a case, when the generator of projective group (16) is admitted, which is not the case for the classical heat equation.

\section{Optimal System OF SUbALGEBRAS OF LiE ALGEBRA OF SYMMETRIES}

For a systematic studying of invariant solutions to diffusion equation (1) we need to construct an optimal system of subalgebras for the admissible Lie algebra of operators. This allows us to exclude equivalent cases from the consideration, that is, the cases reducible one to another by means of the transformations admitted by the equation. Each symmetry of the equation generates an inner automorphism of the Lie algebra of the admitted operators. The optimal system is a set of the representatives from each class formed by elements reducible one to another by the automorphisms of the subalgebras.

We proceed to constructing an optimal system of subalgebras of Lie algebra $L_{9}$ with the basis $X_{1}, \ldots, X_{9}$. We employ a two-step algorithm proposed in [16], see also [7], which is based on using the structure of the algebra. For a three-dimensional nonlinear diffusion equation of an 
integer order with a power coefficient, an optimal system was constructed for a similar system in work [17], but the results can not be easily employed.

The commutators for the algebra $L_{9}$ are given in Table 1

Table 1: Table of commutators

\begin{tabular}{l|lll|lll|l|l|l} 
& $X_{1}$ & $X_{2}$ & $X_{3}$ & $X_{4}$ & $X_{5}$ & $X_{6}$ & $X_{7}$ & $X_{8}$ & $X_{9}$ \\
\hline$X_{1}$ & 0 & 0 & 0 & 0 & $-X_{3}$ & $X_{2}$ & $X_{1}$ & 0 & 0 \\
$X_{2}$ & 0 & 0 & 0 & $X_{3}$ & 0 & $-X_{1}$ & $X_{2}$ & 0 & 0 \\
$X_{3}$ & 0 & 0 & 0 & $-X_{2}$ & $X_{1}$ & 0 & $X_{3}$ & 0 & 0 \\
\hline$X_{4}$ & 0 & $-X_{3}$ & $X_{2}$ & 0 & $-X_{6}$ & $X_{5}$ & 0 & 0 & 0 \\
$X_{5}$ & $X_{3}$ & 0 & $-X_{1}$ & $X_{6}$ & 0 & $-X_{4}$ & 0 & 0 & 0 \\
$X_{6}$ & $-X_{2}$ & $X_{1}$ & 0 & $-X_{5}$ & $X_{4}$ & 0 & 0 & 0 & 0 \\
\hline$X_{7}$ & $-X_{1}$ & $-X_{2}$ & $-X_{3}$ & 0 & 0 & 0 & 0 & 0 & $\frac{2}{\alpha} X_{9}$ \\
\hline$X_{8}$ & 0 & 0 & 0 & 0 & 0 & 0 & 0 & 0 & $\frac{2}{\alpha-1} X_{9}$ \\
\hline$X_{9}$ & 0 & 0 & 0 & 0 & 0 & 0 & $-\frac{2}{\alpha} X_{9}$ & $\frac{2}{1-\alpha} X_{9}$ & 0
\end{tabular}

It is easy to see that the algebra $L_{7}$ with the basis $X_{1}, \ldots, X_{7}$ can be represented as a semi-direct sum of the subalgebra $L_{4}$ and an Abelian ideal $J_{3}$ :

$$
L_{7}=L_{4} \dot{\oplus} J_{3}, \quad L_{4}=\left\{X_{4}, X_{5}, X_{6}, X_{7}\right\}, \quad J_{3}=\left\{X_{1}, X_{2}, X_{3}\right\} .
$$

In the case of the power form of the coefficient $k(u)=u^{\beta}$, the algebra of the admissible operators is extended up to $L_{8}$ :

$$
L_{8}=L_{7} \oplus\left\{X_{8}\right\}
$$

and an one-dimensional subalgebra $\left\{X_{8}\right\}$ is a center of $L_{8}$. And finally, as $\sigma=2 \alpha /(1-\alpha)$, the operator $X_{9}$ is added to the admissible algebra generating its one-dimensional ideal:

$$
L_{9}=L_{8} \dot{\oplus}\left\{X_{9}\right\} .
$$

Each of the operators $X_{i} \in L$ generates an inner automorphism $A_{i}$ of the studied algebra $L$. It can be constructed as a solution to the Cauchy problem

$$
\frac{d \bar{X}}{d a}=\left[X_{i}, \bar{X}\right],\left.\quad \bar{X}\right|_{a=0}=X_{i}
$$

at that, the unknowns are the transformations of the coefficients of the operator $\bar{k}_{i}(a)$ in the original basis: $\bar{X}=\sum_{j=1}^{9} \bar{k}_{j}(a) X_{j}, \quad \bar{k}_{j}(0)=k_{j}$.

It is convenient to write the inner automorphisms for the widest Lie algebra $L_{9}$; for the sake of brevity we provide only changed coefficients:

$$
\begin{aligned}
& A_{1}: \bar{k}_{1}=k_{1}+a_{1} k_{7}, \quad \bar{k}_{2}=k_{2}+a_{1} k_{6}, \quad \bar{k}_{3}=k_{3}-a_{1} k_{5}, \\
& A_{2}: \bar{k}_{1}=k_{1}-a_{1} k_{6}, \quad \bar{k}_{2}=k_{2}+a_{2} k_{7}, \quad \bar{k}_{3}=k_{3}+a_{2} k_{4}, \\
& A_{3}: \bar{k}_{1}=k_{1}+a_{3} k_{5}, \quad \bar{k}_{2}=k_{2}-a_{3} k_{4}, \quad \bar{k}_{3}=k_{3}+a_{3} k_{7}, \\
& A_{4}: \bar{k}_{23}=O_{1} k_{23}, \quad \bar{k}_{56}=O_{1} k_{56}, \\
& A_{5}: \bar{k}_{13}=O_{2} k_{13}, \quad \bar{k}_{46}=O_{2} k_{46}, \\
& A_{6}: \bar{k}_{12}=O_{3} k_{12}, \quad \bar{k}_{45}=O_{3} k_{45} \\
& A_{7}: \bar{k}_{1}=a_{7} k_{1}, \quad \bar{k}_{2}=a_{7} k_{2}, \quad \bar{k}_{3}=a_{7} k_{3}, \quad k_{9}=\left(\frac{1}{a_{7}^{2}}\right)^{1 / \alpha}, a_{7} \neq 0, \\
& A_{8}: \bar{k}_{9}=a_{8} k_{9}, \quad a_{8}>0, \\
& A_{9}: \bar{k}_{9}=k_{9}+\left(\frac{2}{1-\alpha} k_{8}-\frac{2}{\alpha} k_{7}\right) a_{9} .
\end{aligned}
$$


In $A_{4}-A_{6}$ we adopted the following notations for the vector of the coefficients and for the rotation matrix:

$$
k_{i j}=\left(k_{i}, k_{j}\right)^{T}, \quad O_{i}=\left(\begin{array}{rr}
\cos \left(a_{i}\right) & \sin \left(a_{i}\right) \\
-\sin \left(a_{i}\right) & \cos \left(a_{i}\right)
\end{array}\right) .
$$

While constructing an optimal system of subalgebras for the symmetry reduction, to the group of inner automorphisms we add discrete automorphisms generated by reflection of the coordinate axes $x \rightarrow-x, y \rightarrow-y, z \rightarrow-z$; as a result, the initial restriction $a_{7}>0$ in $A_{7}$ is omitted. However, the change $t \rightarrow-t$ influences the integration domain and this is why it is not admitted.

An optimal system of subalgebras is constructed by employing classical algorithms [16], |7] demonstrated in details in [17]: the coordinates of the basis are written as a matrix and are maximally simplified by inner automorphisms, linear transformations and by checking the subalgebra conditions.

An optimal system $\Theta\left(L_{4}\right)$ is known [18] and can be constructed easily:

$$
\begin{array}{ll}
\Theta_{1}\left(L_{4}\right): & 1.1: X_{4}, 1.2: X_{7}, \quad 1.3: X_{4}+\gamma X_{7}, \quad \gamma>0, \\
\Theta_{2}\left(L_{4}\right): & \text { no two-dimensional subalgebras, } \\
\Theta_{3}\left(L_{4}\right): & 3.1: X_{4}, X_{5}, X_{6}, \\
\Theta_{4}\left(L_{4}\right): & 4.1: X_{4}, X_{5}, X_{6}, X_{7} .
\end{array}
$$

To minimize the arbitrariness in the choice of the representatives, one usually impose a normalization condition: together with a subalgebra $K \in \Theta(L)$, the optimal system should also contain its normalizator $\operatorname{Nor}_{L} K$, which is the maximal subalgebra in $L$, for which $K$ is ideal.

In many cases it is convenient to employ cylindrical and spherical coordinate systems for constructing invariant solutions. Hereinafter

$$
R=\sqrt{x^{2}+y^{2}+z^{2}}, \quad r=\sqrt{y^{2}+z^{2}}, \quad r \cos \varphi=y, \quad r \sin \varphi=z .
$$

In Table 2 we provide a constructed optimal system of subalgebras $\Theta\left(L_{7}\right)$; for each subalgebra we give a general form of an invariant solution to equation (1) obtained from the invariants of the subalgebra in the case when the invariants involve $u$.

Table 2: Optimal system $\Theta\left(L_{7}\right)$

\begin{tabular}{c|c|c|c|l} 
no. & Subalgebra & Projection in $L_{4}$ & Nor $_{L_{7}} K$ & Form of invariant solution \\
\hline 1.1 & 4 & - & 3.7 & $v\left(t, x, y^{2}+z^{2}\right)$ \\
1.2 & 7 & - & 4.1 & $v\left(t x^{-2 / \alpha}, t y^{-2 / \alpha}, t z^{-2 / \alpha}\right)$ \\
1.3 & $4+\gamma 7$ & - & 2.1 & $v\left(\gamma \varphi-\ln (r), t x^{-2 / \alpha}, \frac{r}{x}\right)$ \\
1.4 & 1 & 0 & 5.1 & $v(t, y, z)$ \\
1.5 & $1+4$ & 1.1 & 2.1 & $v\left(x-\varphi, y^{2}+z^{2}, t\right)$ \\
\hline 2.1 & 4,7 & - & 2.1 & $v\left(t x^{-2 / \alpha}, \frac{r}{x}\right)$ \\
2.2 & 2,3 & 0 & 5.1 & $v(t, x)$ \\
2.3 & 1,4 & 1.1 & 3.7 & $v(t, r)$ \\
2.4 & 1,7 & 1.2 & 3.7 & $v\left(t y^{-2 / \alpha}, z / y\right)$ \\
2.5 & $1,4+\gamma 7$ & 1.3 & 3.7 & $v\left(t r^{-2 / \alpha}, \gamma \varphi-\ln r\right)$ \\
\hline 3.1 & $4,5,6$ & - & 4.1 & $v(t, R)$ \\
3.2 & $1,2,3$ & 0 & 7.1 & $v(t)$ \\
3.3 & $1+4,2,3$ & 1.1 & 4.2 & $v(t)$ \\
3.4 & $2,3,4$ & 1.1 & 5.1 & $v(t, x)$ \\
3.5 & $2,3,7$ & 1.2 & 4.5 & $v\left(t x^{-2 / \alpha}\right)$ \\
3.6 & $2,3,4+\gamma 7$ & 1.3 & 4.5 & $v\left(t x^{-2 / \alpha}\right)$
\end{tabular}




\begin{tabular}{l|c|c|l|l}
3.7 & $1,4,7$ & 2.1 & 3.7 & $v\left(t r^{-2 / \alpha}\right)$ \\
\hline 4.1 & $4,5,6,7$ & - & 4.1 & $v\left(t R^{-2 / \alpha}\right)$ \\
4.2 & $1,2,3,4$ & 1.1 & 5.1 & $v(t)$ \\
4.3 & $1,2,3,7$ & 1.2 & 7.1 & $c$ \\
4.4 & $1,2,3,4+\gamma 7$ & 1.3 & 5.1 & $c$ \\
4.5 & $2,3,4,7$ & 2.1 & 4.5 & $v\left(t x^{-2 / \alpha}\right)$ \\
\hline 5.1 & $1,2,3,4,7$ & 2.1 & 5.1 & $c$ \\
\hline 6.1 & $1,2,3,4,5,6$ & 3.1 & 7.1 & $v(t)$ \\
\hline 7.1 & $1,2,3,4,5,6,7$ & 4.1 & 7.1 & $c$ \\
\hline
\end{tabular}

We employ a brief writing: $\left\{X_{1}, X_{2}, X_{3}, X_{4}+\gamma X_{7}\right\}$ are denoted as $1,2,3,4+\gamma 7$. We also introduce arbitrary constants $\gamma>0, \lambda \neq 0, \mu \in \mathbb{R}$.

The optimal system $\Theta_{k}\left(L_{8}\right)$ includes subalgebras of two types: all elements $\Theta_{k-1}\left(L_{7}\right)$ with an added operator $X_{8}$, and also subalgebras constructed from $\Theta_{k}\left(L_{7}\right)$ by adding terms with $X_{8}$ to each operator followed by simplification and checking the subalgebra conditions. In Table 3 we list additional elements $\Theta\left(L_{8}\right)$ in comparison with $\Theta\left(L_{7}\right)$.

Table 3: Optimal system $\Theta\left(L_{8}\right)$ as extension of $\Theta\left(L_{7}\right)$

\begin{tabular}{c|c|c|c|l} 
no. & Subalgebra & Projection in $L_{7}$ & Nor $_{L_{8}} K$ & Form of invariant solution \\
\hline 1.6 & 8 & 0 & 8.1 & $t^{-\alpha / \sigma} v(x, y, z)$ \\
1.7 & $4+\gamma 8$ & 1.1 & 4.12 & $e^{\gamma \varphi} v\left(t e^{\sigma \gamma \varphi / \alpha}, x, y^{2}+z^{2}\right)$ \\
1.8 & $7+\lambda 8$ & 1.2 & 5.2 & $x^{\lambda} v\left(t x^{(\sigma \lambda-2) / \alpha}, \frac{y}{x}, \frac{z}{x}\right)$ \\
1.9 & $4+\gamma 7+\mu 8$ & 1.3 & 3.8 & $x^{\frac{\mu}{\gamma}} v\left(t e^{\frac{\sigma \mu-2 \gamma}{\alpha}}, \frac{r}{x}, \gamma \varphi-\ln x\right)$ \\
1.10 & $1+8$ & 1.4 & 5.3 & $t^{-\alpha / \sigma} v\left(t e^{\sigma x / \alpha}, y, z\right)$ \\
1.11 & $1+4+\gamma 8$ & 1.5 & 3.10 & $e^{\gamma \varphi} v\left(t e^{\sigma \gamma \varphi / \alpha}, x-\varphi, y^{2}+z^{2}\right)$ \\
\hline 2.6 & 4,8 & 1.1 & 4.12 & $t^{-\alpha / \sigma} v\left(x, y^{2}+z^{2}\right)$ \\
2.7 & 7,8 & 1.2 & 5.2 & $t^{-\alpha / \sigma} x^{2 / \sigma} v\left(\frac{y}{x}, \frac{z}{x}\right)$ \\
2.8 & $4+\gamma 7,8$ & 1.3 & 3.8 & $t^{-\alpha / \sigma} x^{2 / \sigma} v\left(\gamma \varphi-\ln (r), \frac{r}{x}\right)$ \\
2.9 & 1,8 & 1.4 & 6.2 & $t^{-\alpha / \sigma} v(y, z)$ \\
2.10 & $1+4,8$ & 1.5 & 3.10 & $t^{-\alpha / \sigma} v(r, x-\varphi)$ \\
2.11 & $4,7+\lambda 8$ & 2.1 & 3.8 & $x^{\lambda} v\left(t x^{\frac{\lambda \sigma-2}{\alpha}}, \frac{r}{x}\right)$ \\
2.12 & $4+\gamma 8,7+\mu 8$ & 2.1 & 3.8 & $x^{\mu} e^{\gamma \varphi} v\left(t x^{\frac{\mu \sigma-2}{\alpha}} e^{\frac{\gamma \sigma \phi}{\alpha}}, \frac{r}{x}\right)$ \\
2.13 & $2+8,3$ & 2.2 & 4.7 & $e^{y} v\left(t e^{\frac{\sigma}{\alpha} y}, x\right)$ \\
2.14 & $1,4+\gamma 8$ & 2.3 & 4.12 & $e^{\gamma \varphi} v\left(t e^{\frac{\gamma \sigma}{\alpha} \varphi}, r\right)$ \\
2.15 & $1+8,4+\gamma 8$ & 2.3 & 3.10 & $e^{x+\gamma \varphi} v\left(t e^{\frac{\sigma}{\alpha}(x+\gamma \varphi)}, r\right)$ \\
2.16 & $1,7+\lambda 8$ & 2.4 & 4.12 & $y^{\lambda} v\left(t y^{\frac{\lambda \sigma-2}{\alpha}}, z / y\right)$ \\
2.17 & $1,4+\gamma 7+\lambda 8$ & 2.5 & 4.12 & $r^{\lambda / \gamma} v\left(t r^{\frac{\lambda \sigma-2 \gamma}{\alpha \gamma}}, \gamma \varphi-\ln r\right)$ \\
\hline 3.8 & $4,7,8$ & 2.1 & 3.8 & $x^{2 / \sigma} t^{-\alpha / \sigma} v\left(\frac{r}{x}\right)$ \\
3.9 & $2,3,8$ & 2.2 & 6.2 & $t^{-\alpha / \sigma} v(x)$ \\
3.10 & $1,4,8$ & 2.3 & 4.12 & $t^{-\alpha / \sigma} v(r)$ \\
3.11 & $1,7,8$ & 2.4 & 4.12 & $t^{-\alpha / \sigma} y^{2 / \sigma} v(z / y)$ \\
3.12 & $1,4+\gamma 7,8$ & 2.5 & 4.12 & $t^{-\alpha / \sigma} r^{2 / \sigma} v(\gamma \varphi-\ln r)$ \\
3.14 & $1+8,2,3$ & 3.2 & 5.3 & $e^{x} v\left(t e^{\frac{\sigma x}{\alpha}}\right)$ \\
3.16 & $2,3,4+\gamma 8$ & 5.3 & $e^{\gamma x} v\left(t e^{\frac{\gamma \sigma x}{\alpha}}\right)$ \\
& $2,3,7+\lambda 8$ & 3.5 & 5.6 & $x^{\lambda} v\left(t x^{\frac{\sigma \lambda-2}{\alpha}}\right)$ \\
\hline 15 & & &
\end{tabular}




\begin{tabular}{|c|c|c|c|c|}
\hline 3.17 & $2,3,4+\gamma 7+\lambda 8$ & 3.6 & 5.6 & $x^{\lambda / \gamma} v\left(t x^{\frac{\sigma \lambda-2 \gamma}{\alpha \gamma}}\right)$ \\
\hline 3.18 & $1,4,7+\lambda 8$ & 3.7 & 4.12 & $r^{\lambda} v\left(\operatorname{tr} \frac{\sigma \lambda-2}{\alpha}\right)$ \\
\hline 3.19 & $1,4+\gamma 8,7+\mu 8$ & 3.7 & 4.12 & $e^{\gamma \phi} r^{\mu} v\left(\operatorname{tr}^{\frac{\sigma \mu-2}{\alpha}} e^{\frac{\gamma \sigma \varphi}{\alpha}}\right)$ \\
\hline 4.6 & $4,5,6,8$ & 3.1 & 5.2 & $t^{-\alpha / \sigma} v(R)$ \\
\hline 4.7 & $1,2,3,8$ & 3.2 & 8.1 & $c t^{-\alpha / \sigma}$ \\
\hline 4.8 & $1+4,2,3,8$ & 3.3 & 5.3 & $c t^{-\alpha / \sigma}$ \\
\hline 4.9 & $2,3,4,8$ & 3.4 & 6.2 & $t^{-\alpha / \sigma} v(x)$ \\
\hline 4.10 & $2,3,7,8$ & 3.5 & 5.6 & $c t^{-\alpha / \sigma} x^{2 / \sigma}$ \\
\hline 4.11 & $2,3,4+\gamma 7,8$ & 3.6 & 5.6 & $c t^{-\alpha / \sigma} x^{2 / \sigma}$ \\
\hline 4.12 & $1,4,7,8$ & 3.7 & 4.12 & $c t^{-\alpha / \sigma} r^{2 / \sigma}$ \\
\hline 4.13 & $4,5,6,7+\lambda 8$ & 3.8 & 5.2 & $R^{\lambda} v\left(t R^{\frac{\sigma \lambda-2}{\alpha}}\right)$ \\
\hline 4.14 & $1,2,3,4+\gamma 8$ & 4.1 & 6.2 & $c t^{-\alpha / \sigma}$ \\
\hline 4.15 & $1+8,2,3,4+\gamma 8$ & 4.2 & 5.3 & $c t^{-\alpha / \sigma}$ \\
\hline 4.16 & $1,2,3,7+\lambda 8$ & 4.3 & 8.1 & $c t^{\alpha \lambda /(2-\sigma \lambda)}$ \\
\hline 4.17 & $1,2,3,4+\gamma 7+\lambda 8$ & 4.4 & 6.2 & $c t^{\alpha \lambda /(2 \gamma-\sigma \lambda)}$ \\
\hline 4.18 & $2,3,4,7+\lambda 8$ & 4.5 & 5.6 & $x^{\lambda} v\left(t x^{\frac{\sigma \lambda-2}{\alpha}}\right)$ \\
\hline 4.19 & $2,3,4+\gamma 8,7+\mu 8$ & 4.5 & 5.6 & $c t^{-\alpha / \sigma} x^{2 / \sigma}$ \\
\hline 5.2 & $4,5,6,7,8$ & 4.1 & 5.2 & $c t^{-\alpha / \sigma} R^{2 / \sigma}$ \\
\hline 5.3 & $1,2,3,4,8$ & 4.2 & 6.2 & $c t^{-\alpha / \sigma}$ \\
\hline 5.4 & $1,2,3,7,8$ & 4.3 & 8.1 & - \\
\hline 5.5 & $1,2,3,4+\gamma 7,8$ & 4.4 & 6.2 & - \\
\hline 5.6 & $2,3,4,7,8$ & 4.5 & 5.6 & $c t^{-\alpha / \sigma} x^{2 / \sigma}$ \\
\hline 5.7 & $1,2,3,4,7+\lambda 8$ & 4.6 & 6.2 & $c t^{\alpha \lambda /(2-\sigma \lambda)}$ \\
\hline 5.8 & $1,2,3,4+\gamma 8,7+\mu 8$ & 4.7 & 6.2 & - \\
\hline 6.2 & $1,2,3,4,7,8$ & 5.1 & 6.2 & - \\
\hline 7.2 & $1,2,3,4,5,6,8$ & 6.1 & 8.1 & $c t^{-\alpha / \sigma}$ \\
\hline 7.3 & $1,2,3,4,5,6,7+\lambda 8$ & 7.1 & 8.1 & $c t^{\alpha \lambda /(2-\sigma \lambda)}$ \\
\hline 8.1 & $1,2,3,4,5,6,7,8$ & 7.1 & 8.1 & - \\
\hline
\end{tabular}

Optimal system $\Theta_{k}\left(L_{9}\right)$ is constructed in a same way. We observe that $X_{9}$ corresponds to an ideal but not to the center of the algebra as $X_{8}$. As a result, the term $k_{9} X_{9}$ in one of the basis infinitesimal operator can be excluded by an inner automorphism $A_{9}$ under the conditions

$$
k_{7}^{2}+k_{8}^{2} \neq 0, \quad k_{8} \neq \frac{1-\alpha}{\alpha} k_{7} .
$$

In Table 4 we list subalgebras $\Theta\left(L_{9}\right)$ of the dimension up to 4 not contained in $\Theta\left(L_{8}\right)$.

Table 4: Optimal system $\Theta\left(L_{9}\right)$ as extension of $\Theta\left(L_{8}\right)$

\begin{tabular}{l|l|l} 
no. & Subalgebra & Form of invariant solution \\
\hline 1.12 & 9 & $t^{\alpha-1} v(x, y, z)$ \\
1.13 & $4+9$ & $t^{\alpha-1} v\left(x, r, \frac{t}{1+\varphi t}\right)$ \\
1.14 & $7+\lambda 8$ & $t^{\alpha-1} v\left(y, z, \frac{t}{1+t x}\right)$ \\
1.15 & $1+4+9$ & $t^{\alpha-1} v\left(r, x-\varphi, \frac{t}{1+\varphi t}\right)$ \\
1.16 & $7+\frac{1-\alpha}{\alpha} 8 \pm 9$ & $t^{\alpha-1} x^{\frac{1-\alpha}{\alpha}} v\left(x e^{ \pm \frac{1}{t}}, y e^{ \pm \frac{1}{t}}, z e^{ \pm \frac{1}{t}}\right)$
\end{tabular}




\begin{tabular}{|c|c|c|}
\hline 1.17 & $4+\gamma 7+\frac{1-\alpha}{\alpha} \gamma 8 \pm 9$ & $t^{\alpha-1} x^{\frac{1-\alpha}{\alpha}} v\left(\frac{t}{1 \pm t \gamma^{-1} \ln x}, \gamma \varphi-\ln x, \frac{r}{x}\right)$ \\
\hline 2.18 & 4,9 & $t^{\alpha-1} v(x, r)$ \\
\hline 2.19 & 7,9 & $t^{\alpha-1} x^{\frac{2(1-\alpha)}{\alpha}} v\left(\frac{y}{x}, \frac{z}{x}\right)$ \\
\hline 2.20 & $4+g 7,9$ & $t^{\alpha-1} x^{\frac{2(1-\alpha)}{\alpha}} v\left(\gamma \varphi-\ln x, \frac{r}{x}\right)$ \\
\hline 2.21 & 1,9 & $t^{\alpha-1} v(y, z)$ \\
\hline 2.22 & $1+4,9$ & $t^{\alpha-1} v(r, x-\varphi)$ \\
\hline 2.23 & 8,9 & - \\
\hline 2.24 & $4+\gamma 8,9$ & $t^{\alpha-1} e^{-\gamma \varphi} v(x, r)$ \\
\hline 2.25 & $7+\lambda 8,9$ & $t^{\alpha-1} x^{2 / \alpha-2-\lambda} v\left(\frac{y}{x}, \frac{z}{x}\right)$ \\
\hline 2.26 & $4+\gamma 7+\lambda 8,9$ & $t^{\alpha-1} x^{2 / \alpha-2-\lambda / \gamma} v\left(\frac{r}{x}, \gamma \varphi-\ln x\right)$ \\
\hline 2.27 & $1+8,9$ & $t^{\alpha-1} e^{-x} v(y, z)$ \\
\hline 2.28 & $1+4+\gamma 8,9$ & $t^{\alpha-1} e^{-\gamma x} v(r, x-\varphi)$ \\
\hline 2.29 & $2+9,3$ & $t^{\alpha-1} v\left(x, \frac{t}{1+t y}\right)$ \\
\hline 2.30 & $1,4+9$ & $t^{\alpha-1} v\left(r, \frac{t}{1+t \varphi}\right)$ \\
\hline 2.31 & $1+9,4$ & $t^{\alpha-1} v\left(r, \frac{t}{1+t x}\right)^{\prime}$ \\
\hline 2.32 & $1+9,4+9$ & $t^{\alpha-1} v\left(r, \frac{t}{1+t(x+\varphi)}\right)$ \\
\hline 2.33 & $4,7+\frac{1-\alpha}{\alpha} 8 \pm 9$ & $t^{\alpha-1} x^{\frac{1-\alpha}{\alpha}} v\left(\frac{t}{1 \pm t \ln r}, \frac{t}{1 \pm t \ln x},\right)$ \\
\hline 2.34 & $4+9,7+\frac{1-\alpha}{\alpha} 8+\mu 9$ & $t^{\alpha-1} x^{\frac{1-\alpha}{\alpha}} v\left(\frac{r}{x}, \frac{t}{1+t(\varphi+\mu \ln x)},\right)$ \\
\hline 2.35 & $1,7+\frac{1-\alpha}{\alpha} 8 \pm 9$ & $t^{\alpha-1} r^{\frac{1-\alpha}{\alpha}} v\left(\varphi, \frac{t}{1 \pm t \ln r}\right)$ \\
\hline 2.36 & $1+9,7+\frac{(1-\alpha)(2+\alpha)}{2 \alpha} 8$ & $t^{\alpha-1} r^{\frac{(1-\alpha)(2-\alpha)}{2 \alpha}} v\left(\varphi, \frac{t r}{1+t x}\right)$ \\
\hline 2.37 & $1,4+\gamma 7+\frac{1-\alpha}{\alpha} \gamma 8 \pm 9$ & $t^{\alpha-1} r^{\frac{1-\alpha}{\alpha}} v\left(\frac{t}{1 \pm t \gamma^{-1} \ln r}, \gamma \varphi-\ln r\right)$ \\
\hline 2.38 & $1+9,4+\gamma 7+\frac{(1-\alpha)(2+\alpha)}{2 \alpha} \gamma 8$ & $t^{\alpha-1} r^{\frac{(1-\alpha)(2-\alpha)}{2 \alpha}} v\left(\frac{t r}{1+t x}, \gamma \varphi-\ln r\right)$ \\
\hline 3.21 & $2,3,9$ & $t^{\alpha-1} v(x)$ \\
\hline 3.22 & $1,4,9$ & $t^{\alpha-1} v(r)$ \\
\hline 3.23 & $1,7,9$ & $t^{\alpha-1} y^{\frac{2(1-\alpha)}{\alpha}} v(z / y)$ \\
\hline 3.24 & $1,4+\gamma 7,9$ & $t^{\alpha-1} r^{\frac{2(1-\alpha)}{\alpha}} v(\gamma \varphi-\ln r)$ \\
\hline 3.25 & $4,8,9$ & - \\
\hline 3.26 & $7,8,9$ & - \\
\hline 3.27 & $4+\gamma 7,8,9$ & - \\
\hline 3.28 & $1,8,9$ & - \\
\hline 3.29 & $1+4,8,9$ & - \\
\hline 3.30 & $4,7+\lambda 8,9$ & $t^{\alpha-1} x^{2 / \alpha-2-\lambda} v(r / x)$ \\
\hline 3.31 & $4+\gamma 8,7+\mu 8,9$ & $t^{\alpha-1} e^{-\gamma \varphi} x^{2 / \alpha-2-\mu} v(r / x)$ \\
\hline 3.32 & $2+8,3,9$ & $t^{\alpha-1} e^{-y} v(x)$ \\
\hline 3.33 & $1,4+\gamma 8,9$ & $t^{\alpha-1} e^{-\gamma \varphi} v(r)$ \\
\hline 3.34 & $1+8,4+\gamma 8,9$ & $t^{\alpha-1} e^{-x-\gamma \varphi} v(r)$ \\
\hline 3.35 & $1,7+\lambda 8,9$ & $t^{\alpha-1} y^{2 / \alpha-2-\lambda} v(z / y)$ \\
\hline 3.36 & $1,4+\gamma 7+\lambda 8,9$ & $t^{\alpha-1} r^{2 / \alpha-2-\lambda / \gamma} v(\gamma \varphi-\ln r)$ \\
\hline 3.37 & $1+9,2,3$ & $H^{\alpha-1} v(t / H), H=1+t x$ \\
\hline 3.38 & $1+4+9,2,3$ & $H^{\alpha-1} v(t / H), H=1+t x$ \\
\hline 3.39 & $2,3,4+9$ & $t^{\alpha-1} v(x)$ \\
\hline 3.40 & $2,3,7+\frac{1-\alpha}{\alpha} 8 \pm 9$ & $x^{\frac{1-\alpha}{\alpha}} H^{\alpha-1} v(t / H), H=1 \pm t \ln x$ \\
\hline 3.41 & $2+9,3,7^{\alpha}+\frac{(1-\alpha)(2+\alpha)}{2 \alpha} 8$ & $x^{(1-\alpha)(2+\alpha) /(2 \alpha)} H^{\alpha-1} v(t x / H), H=1+t y$ \\
\hline 3.42 & $2,3,4+\gamma 7+\gamma \frac{1-\alpha}{\alpha} 8 \pm \gamma 9$ & $x^{\frac{1-\alpha}{\alpha}} H^{\alpha-1} v(t / H), H=1 \pm t \ln x$ \\
\hline 3.43 & $1,4,7+\frac{1-\alpha}{\alpha} 8 \pm 9$ & $r^{\frac{1-\alpha}{\alpha}} H^{\alpha-1} v(t / H), H=1 \pm t \ln r$ \\
\hline
\end{tabular}




\begin{tabular}{|c|c|c|}
\hline $\begin{array}{l}3.44: \\
3.45\end{array}$ & $\begin{array}{l}1,4+9,7+\frac{1-\alpha}{\alpha} 8+\mu 9 \\
1+9,4,7+\frac{(1-\alpha)(2+\alpha)}{2 \alpha} 8\end{array}$ & $\begin{array}{l}r^{\frac{1-\alpha}{\alpha}} H^{\alpha-1} v(t / H), H=1+(\mu \ln r+\varphi) t \\
r^{(1-\alpha)(2+\alpha) /(2 \alpha)} H^{\alpha-1} v(\operatorname{tr} / H), H=1+t x\end{array}$ \\
\hline 4.20 & $4,5,6,9$ & $t^{\alpha-1} v(R)$ \\
\hline 4.21 & $1,2,3,9$ & $c t^{\alpha-1}$ \\
\hline 4.22 & $1+4,2,3,9$ & $c t^{\alpha-1}$ \\
\hline 4.23 & $2,3,4,9$ & $c t^{\alpha-1}$ \\
\hline 4.24 & $2,3,7,9$ & $c t^{\alpha-1} x^{\frac{2(1-\alpha)}{\alpha}}$ \\
\hline 4.25 & $2,3,4+\gamma 7,9$ & $c t^{\alpha-1} x^{\frac{2(1-\alpha)}{\alpha}}$ \\
\hline 4.26 & $1,4,7,9$ & $c t^{\alpha-1} r^{\frac{2(1-\alpha)}{\alpha}}$ \\
\hline 4.27 & $4,7,8,9$ & - \\
\hline 4.28 & $2,3,8,9$ & - \\
\hline 4.29 & $1,4,8,9$ & - \\
\hline 4.30 & $1,7,8,9$ & - \\
\hline 4.31 & $1,4+\gamma 7,8,9$ & - \\
\hline 4.32 & $1+8,2,3,9$ & $c t^{\alpha-1} e^{-x}$ \\
\hline 4.33 & $1+4+\gamma 8,2,3,9$ & $c t^{\alpha-1} e^{-\gamma x}$ \\
\hline 4.34 & $2,3,4+\gamma 8,9$ & 一 \\
\hline 4.35 & $2,3,7+\lambda 8,9$ & $c t^{\alpha-1} x^{2 / \alpha-2-\lambda}$ \\
\hline 4.36 & $2,3,4+\gamma 7+\lambda 8,9$ & $c t^{\alpha-1} x^{2 / \alpha-2-\lambda / \gamma}$ \\
\hline 4.37 & $1,4,7+\lambda 8,9$ & $c t^{\alpha-1} r^{2 / \alpha-2-\lambda}$ \\
\hline 4.38 & $1,4+\gamma 8,7+\mu 8,9$ & $c t^{\alpha-1} r^{2 / \alpha-2-\mu} e^{-\gamma \varphi}$ \\
\hline 4.39 & $1,2,3,4+9$ & $c t^{\alpha-1}$ \\
\hline 4.40 & $1+9,2,3,4+9$ & $c t^{\alpha-1}$ \\
\hline 4.41 & $1+9,2,3,4+9$ & $c t^{\alpha-1}$ \\
\hline 4.42 & $4,5,6,7+\frac{1-\alpha}{\alpha} 8 \pm 9$ & $R^{\frac{1-\alpha}{\alpha}} H^{\alpha-1} v(t / H), H=1 \pm t \ln R$ \\
\hline 4.43 & $1,2,3,7+\frac{1-\alpha}{\alpha} 8 \pm 9$ & $c t^{\alpha-1} e^{ \pm \frac{\alpha-1}{\alpha t}}$ \\
\hline 4.44 & $1+9,2,3,7+\frac{(1-\alpha)(2+\alpha)}{2 \alpha} 8$ & $c t^{\frac{(\alpha-1)(\alpha+2)}{2 \alpha}}(1+t x)^{\frac{(1-\alpha)(2-\alpha)}{2 \alpha}}$ \\
\hline 4.45 & $1,2,3,4+\gamma 7+\gamma \frac{1-\alpha}{\alpha} 8 \pm \gamma 9$ & $c t^{\alpha-1} e^{ \pm \frac{\alpha-1}{\alpha t}}$ \\
\hline 4.46 & $\begin{array}{l}1+9,2,3 \\
\quad 4+\gamma 7+\gamma \frac{(1-\alpha)(2+\alpha)}{2 \alpha} 8\end{array}$ & $c t^{\frac{(\alpha-1)(\alpha+2)}{2 \alpha}}(1+t x)^{\frac{(1-\alpha)(2-\alpha)}{2 \alpha}}$ \\
\hline 4.47 & $2,3,4,7+\frac{1-\alpha}{\alpha} 8 \pm 9$ & $x^{\frac{1-\alpha}{\alpha}} H^{\alpha-1} v(t / H), H=1 \pm t \ln x$ \\
\hline 4.48 & $2,3,4+\gamma 9,7+\frac{1-\alpha}{\alpha} 8+\mu 9$ & $c t^{\alpha-1} x^{\frac{1-\alpha}{\alpha}}$ \\
\hline
\end{tabular}

\section{SymmetRY REDUCTIONS}

Subalgebras of small dimensions up to three allow one to construct only invariant solutions of rank two or three, that is, to reduce the equation to a partial differential equation with less number of variables. The resolving of such equations is a complicated problem and in the present work we do not consider it.

As an illustration, we make a symmetry reduction on the subalgebra $2.1\left\{X_{4}, X_{7}\right\}$. Then the corresponding substitution

$$
u=v(\tau, p), \quad \tau=t x^{-2 / \alpha}, \quad p=\frac{y^{2}+z^{2}}{x^{2}}
$$

even to the simplest linear equation $D_{t}^{\alpha} u=u_{x x}+u_{y y}+u_{z z}$ leads us to a rather complicated although linear reduced equation for $v(\tau, p)$ :

$$
D_{\tau}^{\alpha} u=4 p(p+1) v_{p p}+(6 p+4) v_{p}+8 \alpha^{-1} \tau p v_{\tau p}+4 \alpha^{-2} \tau^{2} v_{\tau \tau}+2 \tau \alpha^{-1}\left(1+2 \alpha^{-1}\right) v_{\tau} .
$$

In what follows we consider invariant solutions of rank 1 , for which the symmetry reduction produces ordinary differential equations with integer or fractional derivatives. 
While making symmetry reduction for subalgebras in $\Theta\left(L_{7}\right), \Theta\left(L_{8}\right)$, the most common form of the invariant solution is

$$
u(\mathbf{x}, t)=h(\mathbf{x}) v(\tau), \quad \tau=\operatorname{tg}(\mathbf{x}), \quad \mathbf{x}=(x, y, z)^{T} .
$$

Then by the change of variables in integral (2) we easily get the relation

$$
D_{t}^{\alpha} u=h(\mathbf{x}) g^{\alpha}(\mathbf{x}) D_{\tau}^{\alpha} v(\tau) .
$$

In the particular case

$$
u(\mathbf{x}, t)=v(\tau), \quad \tau=\operatorname{tg}(\mathbf{x}),
$$

after substitution into (1) and in view (20), we get the following form of the reduced equation

$$
g^{\alpha} D_{\tau}^{\alpha} v=\frac{|\nabla g|^{2}}{g^{2}}\left(f v^{\prime \prime}+f^{\prime}\left(v^{\prime}\right)^{2}\right) \tau^{2}+\frac{\Delta g}{g} f v^{\prime} \tau .
$$

We note that employing other equivalent forms of invariant solution can lead to the presence of other fractional derivatives in the reduced equations. For instance, if we choose the form $u=v\left(t^{-\alpha / 2} x\right)$ instead of $u=v\left(t x^{-2 / \alpha}\right)$, the resulting equation involves Erdélyi-Kober operators [8], [19], 20].

Many subalgebras of optimal system lead to the same form of solutions due to the coincidence of the invariants.

The equations arising in constructing invariant solutions of rank 1 are given in Table 5 .

Table 5: Results of symmetry reduction for subalgebras

$\Theta\left(L_{7}\right)$ in the case of arbitrary function $k(u)$

\begin{tabular}{l|l|l} 
Subalgebras & Substitution & Reduced equation \\
\hline $3.2,3.3,4.2,6.1$ & $v(t)$ & $D_{t}^{\alpha} v=0, \quad v=c t^{\alpha-1}$ \\
$3.5,3.6,4.5$ & $v\left(t x^{-2 / \alpha}\right)$ & $D_{\tau}^{\alpha} v=\frac{4}{\alpha^{2}} \tau^{2}\left(k v^{\prime \prime}+\left(v^{\prime}\right)^{2} k^{\prime}\right)+\frac{2(\alpha+2)}{\alpha^{2}} \tau k v^{\prime}$ \\
3.7 & $v\left(t r^{-2 / \alpha}\right)$ & $D_{\tau}^{\alpha} v=\frac{4}{\alpha^{2}} \tau^{2}\left(k v^{\prime \prime}+\left(v^{\prime}\right)^{2} k^{\prime}\right)+\frac{4}{\alpha^{2}} \tau k v^{\prime}$ \\
4.1 & $v\left(t R^{-2 / \alpha}\right)$ & $D_{\tau}^{\alpha} v=\frac{4}{\alpha^{2}} \tau^{2}\left(k v^{\prime \prime}+\left(v^{\prime}\right)^{2} k^{\prime}\right)+\frac{2(2-\alpha)}{\alpha^{2}} \tau k v^{\prime}$
\end{tabular}

For the equation with the power coefficient there are additional forms of reduced equations,

\begin{tabular}{|c|c|c|}
\hline no. & Substitution & Reduced equation \\
\hline 3.8 & $x^{2 / \sigma} t^{-\alpha / \sigma} v(r / x)$ & $\begin{array}{l}\left(\tau^{2}+1\right)\left(v^{\sigma} v^{\prime \prime}+\sigma v^{\sigma-1}\left(v^{\prime}\right)^{2}\right)+\left(\frac{1}{\tau}-2 \tau\left(1+\frac{2}{\sigma}\right)\right) v^{\sigma} v^{\prime}+ \\
\quad+\frac{2}{\sigma}\left(1+\frac{2}{\sigma}\right) v^{\sigma+1}-\frac{\Gamma(1-\alpha / \sigma)}{\Gamma(1-\alpha-\alpha / \sigma)} v=0\end{array}$ \\
\hline $\begin{array}{l}3.9 \\
3.15 \\
4.9\end{array}$ & $t^{-\alpha / \sigma} v(x)$ & $\sigma\left(v^{\prime}\right)^{2}+v v^{\prime \prime}-\frac{\Gamma(1-\alpha / \sigma)}{\Gamma(1-\alpha-\alpha / \sigma)} v^{2-\sigma}=0$ \\
\hline 3.10 & $t^{-\alpha / \sigma} v(r)$ & $v^{\sigma} v^{\prime \prime}+\sigma v^{\sigma-1}\left(v^{\prime}\right)^{2}+\frac{1}{r} v^{\sigma} v^{\prime}-\frac{\Gamma(1-\alpha / \sigma)}{\Gamma(1-\alpha-\alpha / \sigma)} v=0$ \\
\hline 3.11 & $t^{-\alpha / \sigma} y^{2 / \sigma} v(z / y)$ & $\begin{array}{l}\left(1+\frac{1}{\tau^{2}}\right)\left(v^{\sigma} v^{\prime \prime}+\sigma v^{\sigma-1}\left(v^{\prime}\right)^{2}\right)-\left(1+\frac{2}{\sigma}\right) \frac{2}{\tau} v^{\sigma} v^{\prime}+ \\
\quad+\frac{2}{\sigma}\left(1+\frac{2}{\sigma}\right) v^{\sigma+1}-\frac{\Gamma(1-\alpha / \sigma)}{\Gamma(1-\alpha-\alpha / \sigma)} v=0\end{array}$ \\
\hline 3.12 & $t^{-\alpha / \sigma} r^{2 / \sigma} v(h)$ & $\left(\gamma^{2}+1\right)\left(v^{\sigma} v^{\prime \prime}+\sigma v^{\sigma-1}\left(v^{\prime}\right)^{2}\right)-$ \\
\hline 3.13 & $\begin{array}{l}h=\gamma \varphi-\ln r \\
e^{x} v\left(t e^{\sigma x / \alpha}\right)\end{array}$ & $\begin{array}{l}\quad-\frac{4(\sigma+1)}{\sigma^{2}}\left(\sigma v^{\sigma} v^{\prime}-v^{\sigma+1}\right)-\frac{\Gamma(1-\alpha / \sigma)}{\Gamma(1-\alpha-\alpha / \sigma)} v=0 \\
D_{\tau}^{\alpha} v=\frac{\sigma^{2}}{\alpha^{2}} \tau^{2}\left(v^{\sigma} v^{\prime \prime}+\sigma v^{\sigma-1}\left(v^{\prime}\right)^{2}\right)+ \\
\quad+\frac{\sigma^{2}}{\alpha^{2}}(2 \alpha(\sigma+1)+\sigma) \tau v^{\sigma} v^{\prime}+(\sigma+1) v^{\sigma+1}\end{array}$ \\
\hline
\end{tabular}
which are given in Table 6.

Table 6: Results of symmetry reduction for subalgebras $\Theta\left(L_{8}\right)$ in the case $k(u)=u^{\sigma}$ 


\begin{tabular}{|c|c|c|}
\hline 3.14 & $e^{\gamma x} v\left(t e^{\gamma \sigma x / \alpha}\right)$ & $\begin{aligned} D_{\tau}^{\alpha} v & =\frac{\sigma^{2} \gamma^{2}}{\alpha^{2}} \tau^{2}\left(v^{\sigma} v^{\prime \prime}+\sigma v^{\sigma-1}\left(v^{\prime}\right)^{2}\right)+ \\
& +\frac{\sigma \gamma^{2}}{\alpha^{2}}(2 \alpha(\sigma+1)+\sigma) \tau v^{\sigma} v^{\prime}+(\sigma+1) \gamma^{2} v^{\sigma+1}\end{aligned}$ \\
\hline $\begin{array}{l}3.16, \\
4.18\end{array}$ & $x^{\lambda} v\left(t x^{\frac{\sigma \lambda-2}{\alpha}}\right)$ & $\begin{aligned} D_{\tau}^{\alpha} v & =\frac{(\sigma \lambda-2)^{2}}{\alpha^{2}} \tau^{2}\left(v^{\sigma} v^{\prime \prime}+\sigma v^{\sigma-1}\left(v^{\prime}\right)^{2}\right)+ \\
& +\frac{\lambda \sigma-2}{\alpha}\left(2 \lambda(\sigma+1)-1+\frac{\lambda \sigma-2}{\alpha}\right) \tau v^{\sigma} v^{\prime}+\lambda^{2}\left(\sigma+1-\lambda^{-1}\right) v^{\sigma+1}\end{aligned}$ \\
\hline 3.17 & $x^{\lambda / \gamma} v\left(t x^{\frac{\sigma \lambda-2 \gamma}{\alpha \gamma}}\right)$ & $\begin{aligned} D_{\tau}^{\alpha} v & =\frac{(\sigma \lambda-2 \gamma)^{2}}{\alpha^{2} \gamma^{2}} \tau^{2}\left(v^{\sigma} v^{\prime \prime}+\sigma v^{\sigma-1}\left(v^{\prime}\right)^{2}\right)+ \\
& +\frac{\lambda \sigma-2 \gamma}{\alpha \gamma^{2}}\left(2 \lambda(\sigma+1)-\gamma+\frac{\lambda \sigma-2 \gamma}{\alpha}\right) \tau v^{\sigma} v^{\prime}+\frac{\lambda^{2}}{\gamma^{2}}(\sigma+1-\gamma / \lambda) v^{\sigma+1}\end{aligned}$ \\
\hline 3.18 & $r^{\lambda} v\left(t r^{\frac{\sigma \lambda-2}{\alpha}}\right)$ & $\begin{aligned} D_{\tau}^{\alpha} v & =\frac{(\sigma \lambda-2)^{2}}{\alpha^{2}} \tau^{2}\left(v^{\sigma} v^{\prime \prime}+\sigma v^{\sigma-1}\left(v^{\prime}\right)^{2}\right)+ \\
& +\frac{\lambda \sigma-2}{\alpha}\left(2 \lambda(\sigma+1)+\frac{\lambda \sigma-2}{\alpha}\right) \tau v^{\sigma} v^{\prime}+\lambda^{2}(\sigma+1) v^{\sigma+1}\end{aligned}$ \\
\hline 3.19 & $\begin{array}{l}e^{\gamma \phi} r^{\mu} v(t h) \\
h=r^{\frac{\sigma \mu-2}{\alpha}} e^{\frac{\gamma \sigma \varphi}{\alpha}}\end{array}$ & $\begin{aligned} D_{\tau}^{\alpha} v & =\frac{(\sigma \mu-2)^{2}+\gamma^{2} \sigma^{2}}{\alpha^{2}} \tau^{2}\left(v^{\sigma} v^{\prime \prime}+\sigma v^{\sigma-1}\left(v^{\prime}\right)^{2}\right)+\left(\gamma^{2}+\mu^{2}\right)(\sigma+1) v^{\sigma+1} \\
& +\frac{1}{\alpha^{2}}\left(\left(\gamma^{2}+\mu^{2}\right)(2 \alpha \sigma+2 \alpha+\sigma) \sigma+4-4 \mu(\alpha \sigma+\alpha+\sigma)\right) \tau v^{\sigma} v^{\prime}\end{aligned}$ \\
\hline 46 & $t^{-\alpha / \sigma} v(R)$ & $\sigma\left(v^{\prime}\right)^{2}+v v^{\prime \prime}+2 v v^{\prime} / R-\frac{\Gamma(1-\alpha / \sigma)}{\Gamma(1-\alpha-\alpha / \sigma)} v^{2-\sigma}=0$ \\
\hline 4.1 & $R^{\lambda} v\left(t R^{\frac{\sigma \lambda-2}{\alpha}}\right)$ & $\begin{aligned} D_{\tau}^{\alpha} v & =\frac{(\sigma \lambda-2)^{2}}{\alpha^{2}} \tau^{2}\left(v^{\sigma} v^{\prime \prime}+\sigma v^{\sigma-1}\left(v^{\prime}\right)^{2}\right)+ \\
& +\frac{\lambda \sigma-2}{\alpha}\left(2 \lambda(\sigma+1)+1+\frac{\lambda \sigma-2}{\alpha}\right) \tau v^{\sigma} v^{\prime}+\lambda^{2}\left(\sigma+1+\lambda^{-1}\right) v^{\sigma+1}\end{aligned}$ \\
\hline
\end{tabular}

We observe that for all subalgebras containing the operator $X_{8}$, the corresponding solutions are of form $u=t^{-\alpha / \sigma} v(h(x, y, z))$; after the reduction, these solutions produce ordinary differential equations with no fractional derivatives. As it was shown in [12], many of such equations are integrable in quadrature.

All subalgebras containing subalgebra 2.2 (the operators $X_{2}, X_{3}$ ) generate invariant solutions of the one-dimensional nonlinear anomalous diffusion equation. These are, for instance, $3.2-$ 3.6,3.9,3.13 - 3.17. In these cases the reduced equations coincides with ones obtained in work [12].

It is easy to see that in other cases, the reduced equations for invariant solutions of rank 1 are of the form similar to [12]:

$$
D_{\tau}^{\alpha} v=A\left(v^{\sigma} v^{\prime \prime}+\sigma v^{\sigma-1}\left(v^{\prime}\right)^{2}\right)+B \tau v^{\sigma} v^{\prime}+C v^{\sigma+1},
$$

except the cases of reducing to the equations with no fractional derivatives.

We proceed to employing subalgebras $\Theta\left(L_{9}\right)$ not contained in $\Theta\left(L_{8}\right)$. They allow to construct new invariant solutions as $k(u)=u^{\sigma}, \sigma=2 \alpha /(1-\alpha)$.

If such subalgebra contains $X_{9}$ as a basis operator (3.21-3.36, 4.20-4.38), one of the invariants becomes $u t^{\alpha-1}$ and we obtain an analogue of stationary solution $t^{\alpha-1} v(x, y, z)$, for which the left hand side of the equation vanishes. In all other cases, to make a symmetry reduction by combining the invariants of subalgebras we succeed to choose the form of invariant solution

$$
u(\mathbf{x}, t)=h(\mathbf{x})(1+\operatorname{tg}(\mathbf{x}))^{\alpha-1} v(\tau), \quad \tau=\frac{t \lambda(\mathbf{x})}{1+\operatorname{tg}(\mathbf{x})}
$$

and to employ then the relations

$$
\begin{aligned}
& D_{t}^{\alpha} u=\mu(\mathbf{x}) \lambda^{\alpha}(\mathbf{x})(1+\operatorname{tg}(\mathbf{x}))^{-1-\alpha} D_{\tau}^{\alpha} v(\tau) \\
& D_{t}^{\alpha} u=\mu(\mathbf{x}) \lambda^{-1}(\mathbf{x})(\lambda(\mathbf{x})-\tau g(\mathbf{x}))^{1+\alpha} D_{\tau}^{\alpha} v(\tau)
\end{aligned}
$$

obtained by the change of variables in integral (2).

Table 7: Results of symmetry reduction for subalgebras $\Theta\left(L_{9}\right)$ except the solutions of form $t^{\alpha-1} v(\mathbf{x})$ in the case $k(u)=u^{\sigma}, \sigma=2 \alpha /(1-\alpha)$

no. $\quad$ Form of solution and reduced equation 


\begin{tabular}{l|l}
\hline 3.37, & $H^{\alpha-1} v(t / H), H=1+t x$ \\
3.38 & $D_{\tau}^{\alpha} v=\tau^{4}\left(v^{\sigma} v^{\prime \prime}+\sigma v^{\sigma-1}\left(v^{\prime}\right)^{2}\right)+2(\alpha+2) \tau^{3} v^{\sigma} v^{\prime}+(1-\alpha)(\alpha+2) \tau^{2} v^{\sigma+1}$ \\
\hline 3.40, & $\left.x^{\frac{1-\alpha}{\alpha}} H^{\alpha-1} v(t / H)\right), \quad H=1 \pm t \ln x$ \\
3.42, & $D_{\tau}^{\alpha} v=\tau^{4}\left(v^{\sigma} v^{\prime \prime}+\sigma v^{\sigma-1}\left(v^{\prime}\right)^{2}\right)+(\alpha+2)(2 \tau-1 / \alpha) \tau^{2} v^{\sigma} v^{\prime}+$ \\
4.47 & $\quad+\frac{1-\alpha}{\alpha^{2}}((\alpha+2) \alpha \tau(\alpha \tau-1)+1) v^{\sigma+1}$ \\
\hline 3.41 & $x^{(1-\alpha)(2+\alpha) /(2 \alpha)} H^{\alpha-1} v(t x / H), H=1+t y$ \\
& $D_{\tau}^{\alpha} v=\tau^{2}\left(\tau^{2}+1\right)\left(v^{\sigma} v^{\prime \prime}+\sigma v^{\sigma-1}\left(v^{\prime}\right)^{2}\right)+(\alpha+2)\left(2 \tau^{2}+\frac{\alpha+1}{\alpha}\right) \tau^{2} v^{\sigma} v^{\prime}+$ \\
& $\quad+\frac{(1-\alpha)(\alpha+2)}{4 \alpha^{2}}\left(4 \alpha^{2} \tau^{2}+\alpha^{2}+\alpha+2\right) v^{\sigma+1}$ \\
\hline 3.43 & $r^{\frac{1-\alpha}{\alpha}} H^{\alpha-1} v(t / H), \quad H=1 \pm t \ln r$ \\
& $D_{\tau}^{\alpha} v=\tau^{4}\left(v^{\sigma} v^{\prime \prime}+\sigma v^{\sigma-1}\left(v^{\prime}\right)^{2}\right)+2\left((\alpha+2) \tau \mp \frac{\alpha+1}{\alpha}\right) \tau^{2} v^{\sigma} v^{\prime}+$ \\
& $\quad+\frac{1-\alpha}{\alpha^{2}}\left((\alpha+2) \alpha^{2} \tau^{2} \mp 2(\alpha+1) \alpha \tau+\alpha+1\right) v^{\sigma+1}$ \\
\hline 3.44 & $r^{\frac{1-\alpha}{\alpha}} H^{\alpha-1} v(t / H), \quad H=1+(\mu \ln r+\varphi) t$ \\
& $D_{\tau}^{\alpha} v=\left(\mu^{2}+1\right) \tau^{4}\left(v^{\sigma} v^{\prime \prime}+\sigma v^{\sigma-1}\left(v^{\prime}\right)^{2}\right)+2\left(\left(\mu^{2}+1\right)(\alpha+2) \tau-\mu \frac{\alpha+1}{\alpha}\right) \tau^{2} v^{\sigma} v^{\prime}+$ \\
& $\quad+\frac{1-\alpha}{\alpha^{2}}\left(\left(\mu^{2}+1\right)(\alpha+2) \alpha^{2} \tau^{2}-2 \mu(\alpha+1) \alpha \tau+\alpha+1\right) v^{\sigma+1}$ \\
\hline 3.45 & $r^{(1-\alpha)(2+\alpha) /(2 \alpha)} H^{\alpha-1} v(t r / H), \quad H=1+t x$ \\
& $D_{\tau}^{\alpha} v=\tau^{4}\left(v^{\sigma} v^{\prime \prime}+\sigma v^{\sigma-1}\left(v^{\prime}\right)^{2}\right)+\frac{1}{\alpha}\left(2 \alpha(\alpha+2) \tau^{2}+\alpha^{2}+4 \alpha+2\right) \tau v^{\sigma} v^{\prime}+$ \\
& $\quad+\frac{(1-\alpha)(\alpha+2)}{4 \alpha^{2}}\left(4 \alpha^{2} \tau^{2}+(\alpha+1)(\alpha+2)\right) v^{\sigma+1}$ \\
\hline 4.42 & $R^{\frac{1-\alpha}{\alpha}} H^{\alpha-1} v(t / H), \quad H=1 \pm t \ln R$ \\
& $D_{\tau}^{\alpha} v=\tau^{4}\left(v^{\sigma} v^{\prime \prime}+\sigma v^{\sigma-1}\left(v^{\prime}\right)^{2}\right)+\left(2(\alpha+2) \tau \mp \frac{3 \alpha+2}{\alpha}\right) \tau^{2} v^{\sigma} v^{\prime}+$ \\
& $\quad+\frac{1-\alpha}{\alpha^{2}}\left((\alpha+2) \alpha^{2} \tau^{2} \mp(3 \alpha+2) \alpha \tau+2 \alpha+1\right) v^{\sigma+1}$ \\
\hline
\end{tabular}

For Subalgebras 3.37-3.40, 3.42, 4.47 the described solutions are independent of $y, z$; the reduced equations coincide with ones obtained in [12].

We observe that all reduced equations in Table 7 are of the form

$$
D_{\tau}^{\alpha} v=\left(A \tau^{2}+B\right) \tau^{2}\left(v^{\sigma} v^{\prime \prime}+\sigma v^{\sigma-1}\left(v^{\prime}\right)^{2}\right)+\left(C \tau^{2}+D \tau+E\right) \tau v^{\sigma} v^{\prime}+\left(F \tau^{2}+G \tau+H\right) v^{\sigma+1} .
$$

Invariant solutions of rank 0 are constructed on the base of subalgebras of dimension 4 and greater. According to Tables 2, 3, 4, most part of such solutions are power functions. They also correspond to exact power solutions of reduced equations for solutions of rank 1.

\section{CONCLUSION}

The group classification made in the present work and the procedure of symmetry reduction demonstrates the possibility of applying classical algorithms of the group analysis of differential equations to a systematic study of nonlinear equations with fractional derivatives and several independent variables. The results agree with earlier results for the one-dimensional model.

The constructed optimal systems of subalgebras and the forms of invariant solutions can be applicable for other three-dimensional models with fractional derivatives. We succeed to avoid main difficulties of constructing reduced equations like changing the limits and a type of the operator of fractional differentiation by choosing an appropriate form of the invariant solution.

\section{BIBLIOGRAPHY}

1. St.G. Samko, A.A. Kilbas, O.I. Marichev. Fractional integrals and derivatives: theory and applications. Nauka i Tekhnika, Minsk (1987). [Gordon and Breach, New York (1993).]

2. A.A. Kilbas, H.M. Srivastava, J.J. Trujillo. Theory and applications of fractional differential equations. Elsevier, Amsterdam (2006).

3. V.V. Uchaikin. Method of fractional derivatives. Artishok, Ulyanovsk (2008). (in Russian).

4. L. Caffarelli, J.L. Vazquez. Nonlinear porous medium flow with fractional potential pressure // Arch. Rat. Mech. Anal. 202:2, 537-565 (2011).

5. Płociniczak Ł. Analytical studies of a time-fractional porous medium equation. Derivation, approximation and applications // Comm. Nonl. Sci. Numer. Simul. 24:1-3, 169-183 (2015). 
6. L.V. Ovsiannikov. Group analysis of differential equations. Nauka, Moscow (1978). [Academic Press, New York (1982).]

7. S.V. Khabirov, Yu.A. Chirkunov. Emelents of symmetry analysis of differential equations of contionuous media mechanics. Novosibirsk State Tech. Univ., Novosibirsk (2012). (in Russian).

8. R.K. Gazizov, A.A. Kasatkin, S.Yu. Lukashchuk. Symmetries, conservation laws and group invariant solutions of fractional PDEs // in "Fractional Differential Equations", De Gruyter, Berlin, 353-382 (2019).

9. R.K. Gazizov, S.Yu. Lukashchuk Fractional-differential approach to the modelling of filtration processes in complicated inhomogeneous porous media // Vestnik UGATU. 21:4(78), 104-112 (2017). (in Russian).

10. V.A. Dorodnitsyn, I.V. Knyazeva, S.R. Svirshchevskij. Group properties of the heat-conduction equation with a source in the two- and three-dimensional cases // Diff. Uravn. 19:7, 1215-1223 (1983). [Diff. Equat. 19:7, 901-908 (1983).]

11. N.H. Ibragimov CRC Handbook of Lie group analysis of differential equations. Vol.1. Symmetries, exact solutions and conservation laws. CRC Press Inc., Boca Raton, Florida (1994).

12. S. Yu. Lukashchuk. Symmetry reduction and invariant solutions for nonlinear fractional diffusion equation with a source term // Ufimskij Matem. Zhurn. 8:4, 114-126 (2016). [Ufa Math. J. 8:4, 111-122 (2016).]

13. E. Lashkarian, S.R. Hejazi, E. Dastranj. Conservation laws of $(3+\alpha)$-dimensional time-fractional diffusion equation // Comput. Math. Appl. 75:3, 740-754 (2018).

14. S.Y. Lukashchuk, A.V. Makunin. Group classification of nonlinear time-fractional diffusion equation with a source term // Appl. Math. Comput. 257, 335-343 (2015).

15. S.Yu. Lukaschuk. On one class of systems of fractional differential equations with symmetries of only linear autonomous form // in Book of Abstracts of International Scientific Conference "Ufa Autumn Mathematical School", BSU Publ., Ufa, 134-136 (2019). (in Russian).

16. L.V. Ovsyannikov. On optimal systems of subalgebras // Dokl. AN. 333:6, 702-704 (1993). [Dokl. Math. 48:3, 645-649 (1994).]

17. A.M. Ilyasov. Optimal system of Lie algebra subalgebras of the point symmetries group for nonlinear heat equation without source // Ufimskij Matem. Zhurn. 5:3, 54-66 (2013). [Ufa Math. J. 5:3, 53-66 (2013).]

18. J. Patera, P. Winternitz. Subalgebras of real three-and four-dimensional Lie algebras // J. Math. Phys. 18:7, 1449-1455 (1977).

19. E. Buckwar, Y. Luchko. Invariance of a partial differential equation of fractional order under the Lie group of scaling transformations // J. Math. Anal. Appl. 227:1, 81-97 (1998).

20. R. Sahadevan, T. Bakkyaraj. Invariant analysis of time fractional generalized Burgers and Korteweg-de Vries equations // J. Math. Anal. Appl. 393:2, 341-347 (2012).

Rafail Kavyevich Gazizov,

Ufa State Avaition Technical University,

Karl Marx str. 12,

450008, Ufa, Russia

E-mail: gazizovrk@gmail.com

Alexei Alexandrovich Kasatkin,

Ufa State Avaition Technical University,

Karl Marx str. 12,

450008, Ufa, Russia

E-mail: kasatkin@ugatu.su

Stanislav Yurievich Lukaschuk,

Ufa State Avaition Technical University,

Karl Marx str. 12,

450008, Ufa, Russia

E-mail: Isu@ugatu.su 